\title{
Synthesis of 3-Alkenyl-1-azaanthraquinones via Diels-Alder and Electron Transfer Reactions
}

\section{Pascal Rathelot, Vincent Rémusat and Patrice Vanelle*}

Laboratoire de Chimie Organique, UMR-CNRS 6517, Faculté de Pharmacie, 27 Boulevard Jean Moulin, 13385 Marseille Cx 5, France.

* Author to whom correspondence should be addressed; E-mail: patrice.vanelle@ pharmacie.univ-mrs.fr

Received: 8 November 2002; in revised form: 16 December 2002 / Accepted: 17 December 2002 / Published: 31 December 2002

\begin{abstract}
A convenient route to 3-alkenyl-1-azaanthraquinones via a hetero Diels-Alder reaction between an azadiene and naphthoquinone, a free radical chlorination and an electron transfer reaction is reported.
\end{abstract}

Keywords: Naphthoquinone, azadiene, azaanthraquinone, Diels-Alder, $\mathrm{S}_{\mathrm{RN}} 1$.

\section{Introduction}

The anthracyclines (doxorubicin, daunorubicin) play an important role in the treatment of many human lymphomas, leukemia and solid tumors [1, 2]. However, their clinical use is limited mainly by two major problems, namely their cumulative cardiotoxicity and the appearance of intrinsic and acquired drug resistance. The search for new analogues having better therapeutic efficacy led us to synthesize 3-alkenyl-1azaanthraquinone derivatives.

\section{Results and Discussion}

The first step proceeds via a hetero Diels-Alder reaction between an azadiene and naphthoquinone. Various groups have studied such a reaction, including its regioselectivity when it was performed using 
unsymmetrical quinones [3]. In this case the [4 + 2] cycloaddition between 1-dimethylamino-3-methyl-1-aza1,3-butadiene and naphthoquinone was followed by elimination of dimethylamine and oxidation to afford the aromatized 3-methyl-1-azaanthraquinone (2) (Scheme 1).

\section{Scheme 1.}

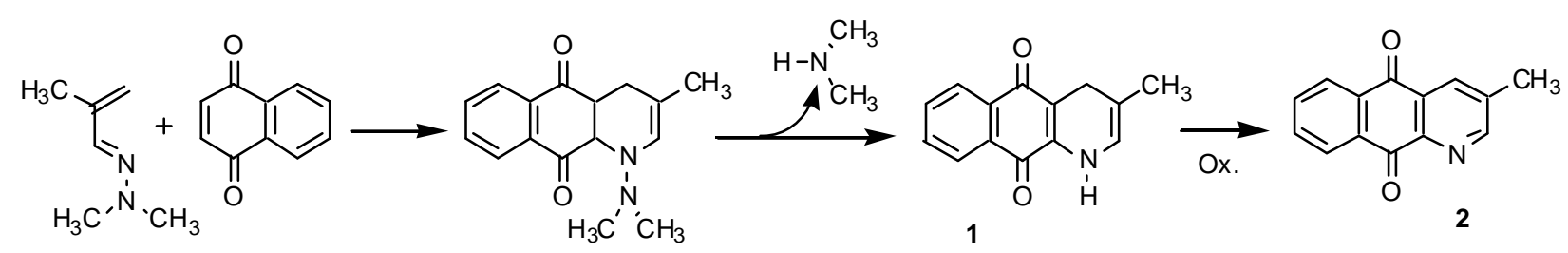

By performing the reaction in acetonitrile at room temperature for 24 hours and using 2 equivalents of naphthoquinone, Serckx-Poncin [4] obtained, in an one pot synthesis, the aromatized adduct $\mathbf{2}$ in $92 \%$ yield. Lee [5] modified this process by replacing the excess naphthoquinone with stirring in the presence of silica gel to effect the oxidation. Compound 2 was then obtained in $51 \%$ yield. Using the Serckx-Poncin procedure with a slight excess (1.2 eq.) of napthoquinone we only obtained compound 1 in 55\% yield, resulting from the cycloaddition followed by the elimination of dimethylamine, instead of the 3-methyl-1-azaanthraquinone (2). Oxidation was performed by stirring in refluxing ethanol as described for the reaction with 5hydroxynaphthoquinone [6]. Derivative 2 was isolated in 95\% yield after purification by chromatography. In order to optimize this reaction we have treated directly the crude residue, after acetonitrile removal, with boiling ethanol. A simple recrystallization from ethanol led to $\mathbf{2}$ in $90 \%$ yield. The advantage of such a procedure lies in the fact that you don't have to use a 2 eq. excess of naphthoquinone, in one hand and you can obtain 2 without any purification by column chromatography, on the other hand.

The 3-methyl group was then halogenated according to the Newkome procedure [7]. (Scheme 2). Various experimental conditions, using $N$-chlorosuccinimide as reactant, were tried in an attempt to increase the yield of the reaction. The best yield of $\mathbf{3}$ was $31 \%$ along with the starting material 2 (47\%) and trace amounts of the dihalogenated derivative were also isolated.

Scheme 2.

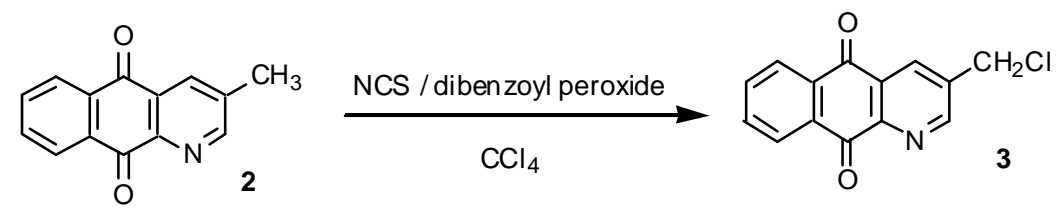

3-Chloromethyl-1-azaanthraquinone (3) was then reacted with the lithium salts of 2-nitropropane or 2,2dimethyl-5-nitro-1,3-dioxane via an $\mathrm{S}_{\mathrm{RN}} 1$ mechanism. Using Kornblum's conditions [8,9] in degassed DMF, only the ethylenic derivative, resulting from by a base promoted nitrous acid elimination of the corresponding $C$-alkylated product, was isolated in good yield (Scheme 3). 


\section{Scheme 3.}

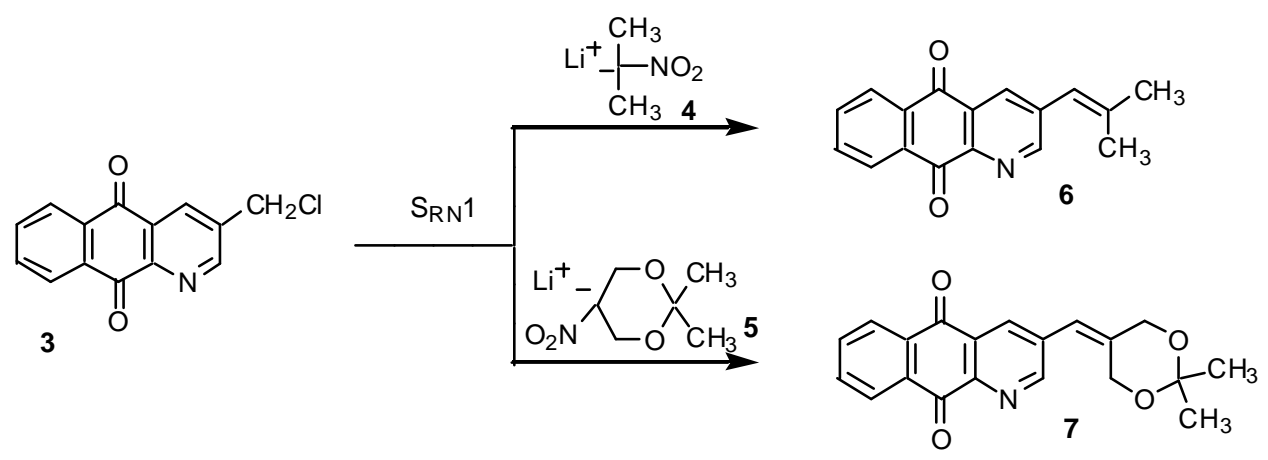

\section{Conclusions}

A first key-step has been optimized, providing 3-methyl-1-azaanthraquinone (2) in high yield after an easier work-up than those previously described in the literature. Thus, this work has shown that such a pathway is a convenient route for the synthesis of 3-alkenyl-1-azaanthraquinones and it is currently being extended to various anions to produce a new series of azaanthraquinones which will be assessed for biological activity towards various tumor cell lines.

\section{Acknowledgments}

We are grateful to the Centre National de la Recherche Scientifique for its financial support. We also wish to express our thanks to Gilles Lanzada for his technical collaboration.

\section{Experimental}

\section{General}

Melting points were determined on a Büchi B540 apparatus and are uncorrected. Elemental analyses were performed by the Centre de Microanalyses of the University of Aix-Marseille 3. Both ${ }^{1} \mathrm{H}$ - and ${ }^{13} \mathrm{C}$ NMR spectra were determined on a Bruker ARX 200 spectrometer. The ${ }^{1} \mathrm{H}$ - chemical shifts were reported as parts per million downfield from tetramethylsilane $\left(\mathrm{Me}_{4} \mathrm{Si}\right)$ and the ${ }^{13} \mathrm{C}$ - chemical shifts were referenced to the $\mathrm{CDCl}_{3}$ solvent peak (76.9 ppm). Silica gel 60 (Merck, 230-400 mesh) was used for column chromatography. Thin-layer chromatography was performed with silica gel Merck 60F-254 (0.25 mm layer thickness).

Synthesis of 3-methyl-1-azaanthraquinone (2).

Naphthoquinone (1 g, $32.2 \mathrm{mmol}$ ) was added to a solution of 1-dimethylamino-3-methyl-1-aza-1,3butadiene $(3 \mathrm{~g}, 26.8 \mathrm{mmol})$ in acetonitrile $(120 \mathrm{~mL})$. The reaction mixture, which rapidly turned dark red, was 
stirred for $20 \mathrm{~h}$ at room temperature. Then, acetonitrile was removed under reduced pressure, the crude residue was dissolved in ethanol $(110 \mathrm{~mL})$ and the reaction mixture was stirred for $20 \mathrm{~h}$ in refluxing ethanol. After cooling at room temperature, it was evaporated and recrystallized from ethanol, to afford $5.38 \mathrm{~g}(90 \%$ yield) of 2 as yellow needles, m.p. $244{ }^{\circ} \mathrm{C} ;{ }^{1} \mathrm{H}-\mathrm{NMR}\left(\mathrm{CDCl}_{3}\right) \delta 2.50\left(\mathrm{~s}, 3 \mathrm{H}, \mathrm{CH}_{3}\right), 7.68-7.81\left(\mathrm{~m}, 2 \mathrm{H}, \mathrm{H}_{6,7}\right)$, 8.13-8.24 (m, $1 \mathrm{H}, \mathrm{H}_{5}$ or 8$), 8.26-8.39\left(\mathrm{~m}, 2 \mathrm{H}, \mathrm{H}_{4}, \mathrm{H}_{5}\right.$ or 8$), 8.85\left(\mathrm{~d}, 1 \mathrm{H}, \mathrm{J}=2.0 \mathrm{~Hz}, \mathrm{H}_{2}\right) .{ }^{13} \mathrm{C} \mathrm{NMR}\left(\mathrm{CDCl}_{3}\right)$ $\delta 18.92\left(\mathrm{CH}_{3}\right), 127.21(\mathrm{CH}), 127.88(\mathrm{CH}), 130.16(\mathrm{C}), 132.81(\mathrm{C}), 133.50(\mathrm{C}), 134.39(\mathrm{CH}), 134.67$ $(\mathrm{CH}), 135.05(\mathrm{CH}), 138.92(\mathrm{C}), 146.84(\mathrm{C}), 155.83(\mathrm{CH}), 181.57(\mathrm{C}), 183.04(\mathrm{C})$.

Free radical chlorination of 2 : preparation of 3-chloromethyl-1-azaanthraquinone (3).

2 (3 g, 13,4 mmol), $N$-chlorosuccinimide $(3.63 \mathrm{~g}, 27.18 \mathrm{mmol})$ and a catalytic amount of dibenzoyl peroxide were stirred in refluxing carbon tetrachloride $(200 \mathrm{~mL})$ for $2 \mathrm{~h}$ in the presence of two $60 \mathrm{~W}$ tungsten lamps. After cooling to room temperature, the solvent was removed under reduced pressure. The crude residue was purified by chromatography on a silica gel column, eluting with ethyl acetate, to yield $1.07 \mathrm{~g}$ (31\%) of 3-chloromethyl-1-azaanthraquinone (3) as brown crystals, m.p. $160{ }^{\circ} \mathrm{C}$; Rf: 0.75 (ethyl acetate). IR $(\mathrm{KBr}) \mathrm{cm}^{-1}: 1680,1585 .{ }^{1} \mathrm{H}-\mathrm{NMR}\left(\mathrm{CDCl}_{3}\right) \delta 4.75\left(\mathrm{~s}, 2 \mathrm{H}, \mathrm{CH}_{2} \mathrm{Cl}\right), 7.80-7.88\left(\mathrm{~m}, 2 \mathrm{H}, \mathrm{H}_{6,7}\right), 8.26-8.33(\mathrm{~m}$, $1 \mathrm{H}, \mathrm{H}_{5}$ or 8 ), 8.34-8.42 (m, $1 \mathrm{H}, \mathrm{H}_{5}$ or 8$), 8.64\left(\mathrm{~d}, 1 \mathrm{H}, \mathrm{J}=2.1 \mathrm{~Hz}, \mathrm{H}_{4}\right), 9.09$ (d, $\left.1 \mathrm{H}, \mathrm{J}=2.1 \mathrm{~Hz}, \mathrm{H}_{2}\right) ;{ }^{13} \mathrm{C}-$ NMR $\left(\mathrm{CDCl}_{3}\right) \delta 42.35\left(\mathrm{CH}_{2}\right), 126.86(\mathrm{CH}), 127.22(\mathrm{CH}), 130.42(\mathrm{C}), 132.79(\mathrm{C}), 133.66(\mathrm{C}), 134.72$ $(\mathrm{CH}), 134.79(\mathrm{CH}), 135.02(\mathrm{CH}), 138.41(\mathrm{C}), 148.35(\mathrm{C}), 154.71(\mathrm{CH}), 180.89(\mathrm{C}), 182.57(\mathrm{C})$; Anal. Calcd for $\mathrm{C}_{14} \mathrm{H}_{8} \mathrm{ClNO}_{2}$ (257.67): C, 65.26; H, 3.13; N, 5.44\%. Found: C, 65.19; H, 3.22; N, 5.37\%.

\section{Preparation of nitronate lithium salts $\mathbf{4}$ and $\mathbf{5}$.}

The synthesis of the lithium salts of 2-nitropropane and 2,2-dimethyl-5-nitro-1,3-dioxane (4 and 5) has been previously described [10].

$S_{R N} 1$ reactions of chloride 3 with the lithium salts 4 and 5: preparation of 3-(2-methylpropenyl)benzo[g]quinoline-5,10-dione (6) and 3-(2,2-dimethyl-[1,3]dioxan-5-ylidenemethyl)-benzo[g]quinoline-5,10-dione (7).

To a solution of 2-nitropropane lithium salt (4) $(370 \mathrm{mg}, 3.88 \mathrm{mmol}$ ) or 2,2-dimethyl-5-nitro-1,3-dioxane lithium salt (5) in dry DMF $(10 \mathrm{~mL})$, the chloride $3(250 \mathrm{mg}, 0.97 \mathrm{mmol})$ was added under nitrogen and anhydrous conditions. The reaction mixture was irradiated with two $60 \mathrm{~W}$ tungsten lamps and stirred at room temperature for $20 \mathrm{~h}$. Then, it was poured into water $(100 \mathrm{~mL})$. The aqueous solution was extracted with dichloromethane $(3 \times 50 \mathrm{~mL})$. The organic extracts were washed with water $(6 \times 300 \mathrm{~mL})$, dried over anhydrous $\mathrm{Na}_{2} \mathrm{SO}_{4}$ and evaporated under vacuum. For the reaction with 4 , the crude residue was purified by chromatography on a silica gel column eluting with ethyl acetate. After recrystallization from isopropanol, 190 
$\mathrm{mg}$ (75\% yield) of the ethylenic derivative 3-(2-methyl-propenyl)-benzo[g]quinoline-5,10-dione (6) was obtained as a brown solid, m.p. $137{ }^{\circ} \mathrm{C}$; Rf: 0.77 (ethyl acetate). IR $(\mathrm{KBr}) \mathrm{cm}^{-1}: 2900,1680,1570 .{ }^{1} \mathrm{H}-$ NMR $\left(\mathrm{CDCl}_{3}\right) \delta 1.97\left(\mathrm{~s}, 3 \mathrm{H}, \mathrm{CH}_{3}\right), 2.00\left(\mathrm{~s}, 3 \mathrm{H}, \mathrm{CH}_{3}\right), 6.35$ (broad s, 1H, ethylenic $\left.\mathrm{H}\right), 7.75-7.88(\mathrm{~m}, 2 \mathrm{H}$, $\left.\mathrm{H}_{6,7}\right), 8.23-8.31\left(\mathrm{~m}, 1 \mathrm{H}, \mathrm{H}_{5}\right.$ or 8$), 8.35-8.43\left(\mathrm{~m}, 2 \mathrm{H}, \mathrm{H}_{4}, \mathrm{H}_{5}\right.$ or 8$), 8.91\left(\mathrm{~d}, 1 \mathrm{H}, \mathrm{J}=2.1 \mathrm{~Hz}, \mathrm{H}_{2}\right) ;{ }^{13} \mathrm{C} \mathrm{NMR}$ $\left(\mathrm{CDCl}_{3}\right) \delta 19.83\left(\mathrm{CH}_{3}\right), 27.34\left(\mathrm{CH}_{3}\right), 120.53(\mathrm{CH}), 127.09(\mathrm{CH}), 127.73(\mathrm{CH}), 130.02(\mathrm{C}), 132.76(\mathrm{C})$, $133.48(\mathrm{C}), 133.54(\mathrm{CH}), 134.24(\mathrm{CH}), 134.57(\mathrm{CH}), 138.75(\mathrm{C}), 143.15(\mathrm{C}), 145.90(\mathrm{C}), 155.07(\mathrm{CH})$, 181.28 (C), 182.94 (C); Anal. Calcd for $\mathrm{C}_{17} \mathrm{H}_{13} \mathrm{NO}_{2}$ (263.29): C, 77.55; H, 4.98; N, 5.32\%. Found: C, 77.59; H, 4.96; N, 5.18\%.

For the reaction with $\mathbf{5}$, the crude residue was directly recrystallized from isopropanol yielding $230 \mathrm{mg}$ (71\%) of 3-(2,2-dimethyl-[1,3]dioxan-5-ylidenemethyl)-benzo[g]quinoline-5,10-dione (7) as a brown solid, m.p. $169{ }^{\circ} \mathrm{C}$; IR $(\mathrm{KBr}) \mathrm{cm}^{-1}$ : 2980, 1680, 1575. ${ }^{1} \mathrm{H}-\mathrm{NMR}\left(\mathrm{CDCl}_{3}\right) \delta 1.45\left(\mathrm{~s}, 6 \mathrm{H}, 2 \mathrm{CH}_{3}\right), 4.46($ broad s, $2 \mathrm{H}, \mathrm{CH}_{2} \mathrm{O}$ ), 4.69 (broad s, $2 \mathrm{H}, \mathrm{CH}_{2} \mathrm{O}$ ), 6.39 (broad s, $1 \mathrm{H}$, ethylenic $\mathrm{H}$ ), 7.80-7.88 (m, 2H, $\left.\mathrm{H}_{6,7}\right), 8.25$ $8.32\left(\mathrm{~m}, 2 \mathrm{H}, \mathrm{H}_{4}, \mathrm{H}_{5}\right.$ or 8$), 8.35-8.43\left(\mathrm{~m}, 1 \mathrm{H}, \mathrm{H}_{5}\right.$ or 8$), 8.85\left(\mathrm{~d}, 1 \mathrm{H}, \mathrm{J}=2.1 \mathrm{~Hz}, \mathrm{H}_{2}\right) ;{ }^{13} \mathrm{C}-\mathrm{NMR}\left(\mathrm{CDCl}_{3}\right) \delta$ $23.78\left(2 \mathrm{CH}_{3}\right), 60.53\left(\mathrm{CH}_{2}\right), 64.06\left(\mathrm{CH}_{2}\right), 99.87(\mathrm{C}), 117.55(\mathrm{CH}), 127.24(\mathrm{CH}), 127.88(\mathrm{CH}), 130.08$ (C), $132.70(\mathrm{C}), 133.47(\mathrm{C}), 133.68(\mathrm{CH}), 134.47(\mathrm{CH}), 134.81(\mathrm{CH}), 136.08(\mathrm{C}), 144.36(\mathrm{C}), 146.64$ (C), $154.60(\mathrm{CH}), 181.09(\mathrm{C}), 182.62(\mathrm{C})$; Anal. Calcd for $\mathrm{C}_{20} \mathrm{H}_{17} \mathrm{NO}_{4}$ (335.35): C, 71.63; H, 5.11; N, 4.18\%. Found: C, 71.50; H, 5.18; N, 4.09\%.

\section{References}

1. Arcamone, F. Cancer Res. 1985, 45, 5995-5999.

2. Weiss, R. B.; Sarosy, G.; Clagett-Carr, K.; Russo, M.; Leyland-Jones, B. Cancer Chemother. Pharmacol. 1986, 18, 185-197.

3. Pautet, F.; Nebois, P.; Bouaziz, Z.; Fillion, H. Heterocycles, 2001, 54, 1095-1138.

4. Serckx-Poncin, B.; Hesbain-Frisque, A.-M.; Ghosez, L. Tetrahedron Lett. 1982, 23, 3261-3264.

5. Lee, H.; Hong, S. -S.; Kim, Y. -H. Bioorg. Med. Chem. Lett. 1996, 6, 933-936.

6. Potts, K. T.; Bhattachargee, D.; Walsh, E. B. J. Chem. Soc., Chem. Commun. 1984, 114-116.

7. Newkome, G. R.; Kieffer, G. E.; Xia, Y. -J. Synthesis 1984, 8, 676-679.

8. Kornblum, N.; Pink, P. Tetrahedron 1963, 19, 17-22.

9. Kornblum, N.; Carlson, S. C.; Widmer, J.; Fifolt, M. J.; Newton, B. N.; Smith, R. G. J. Org. Chem. 1978, 43, 1394-1399.

10. Vanelle, P.; Madadi, N.; Roubaud, C.; Maldonado, J.; Crozet, M. P. Tetrahedron, 1991, 47, 51735184.

Samples Availability: Available from the authors.

(C) 2002 by MDPI (http://www.mdpi.org). Reproduction is permitted for noncommercial purposes. 